First publ. in: Fungal Genetics and Biology 24 (1998), pp. 77-85

\title{
Endocytosis and Membrane Turnover in the Germ Tube of Uromyces fabae
}

\author{
J ochen Hoffmann and Kurt Mendgen \\ Lehrstuhl Phytopathologie, Fakultät für B iologie, Universität Konstanz, 78457 Konstanz, Germany
}

Accepted for publication May 8, 1998

Hoffmann, J . and Mendgen, K. 1998. Endocytosis and membrane turnover in the germ tube of Uromyces fabae. Fungal Genetics and Biology 24, 77-85. We have used the fluorescent dye FM 4-64 as a tracer to demonstrate bulk membrane internalization (endocytosis) and redistribution of the dye within the cytoplasm of the germ tube of the rust fungus Uromyces fabae. Staining of the hyphal membrane was detected $4 \mathrm{~s}$ after application of FM 4-64 and reached a maximum after $1 \mathrm{~min}$. The highest fluorescence intensity occurred in the apex. Subsequently, staining of the plasma membrane decreased and a subapical region of the fungal protoplast (5-20 $\mu \mathrm{m}$ from the tip) displayed increasing fluorescence with a maximum after 5 min. Fluorescence in the subapical region was redistributed to an area in the hyphal tip, which corresponds to the accumulation of apical vesicles, after 10-15 min and subsequently to a cytoplasmic region in front of the two nuclei (35-45 $\mu \mathrm{m}$ from the tip). We conclude from our measurements of membrane fluorescence that the turnover time from endocytosis to secretion of the dye amounts to $15 \mathrm{~min}$. The uptake of the dye into the cytoplasm, but not membrane loading, could be inhibited completely with $5 \mathrm{mM} \mathrm{NaN}_{3}$ or by a temperature shift to $4^{\circ} \mathrm{C}$. This is the first evidence for endocytosis in a fungal germ tube. 1998 Academic Press

Index Descriptors: tip growth; hyphal apex; Spitzenkörper; apical vesicle cluster.

Extension of the hyphal tip appears to be sustained by a massive flow of vesicles from behind. Collinge and Trinci (1974) calculated and estimated some 38,000 vesicles per minute which seemed to support the extension of a N eurospora hypha growing at a speed of $35 \mu \mathrm{m} / \mathrm{min}$. After releasing their contents, the membranes of the vesicles contribute to the extension of the plasma membrane at the hyphal tip. Endocytotic traffic may counterbalance a possible increase of membrane surface and maintain an appropriate size and protein and lipid content of the plasma membrane (Gow, 1989). Although endocytotic traffic can occur by several independent pathways (Riezman, 1997), a direct proof of endocytosis is difficult. E lectron micrographs do not show convincingly any endocytotic events in growing hyphae. In Dictyostelium (H enkel et al., 1996) and Saccharomyces cer evisiae (Vida and Emr, 1995) FM 4-64 and similar fluorescent lipophilic styryl dyes have been used to monitor endocytosis. F M 4-64 labeled bulk transport from the plasma membrane to the vacuole. Endocytotic intermediates, presumably endosomes, were demonstrated on their way to the vacuole.

The organization of a germ tube of a rust fungus is especially suited for studies of vectorial transport of cytoplasmic components. A continued forward migration of the cytoplasm occurs as the germ tube grows. Within the germ tube walls, the cytoplasm leaves behind one or more vacuoles which occupy almost the entire volume of the older part of the hypha (H eath and $\mathrm{H}$ eath, 1979).

Starting from the apex, $\mathrm{H}$ och and Staples (1983) defined five regions of cytoplasmic organization of the actively growing germling. Region I constitutes the first 15-20 $\mu \mathrm{m}$ of the hyphal tip. It includes an accumulation of apical vesicles in the extreme apex followed by an abundance of Golgi equivalents and mitochondria. Region II, covering the next 20-25 $\mu \mathrm{m}$, includes Golgi equivalents and mitochondria and a network of small vacuoles, most of which 
appear interconnected with each other and oriented in the longitudinal axis of the hypha. Region III is characterized by the presence of two nuclei and regions I V and $\mathrm{V}$ by lipid bodies, filamentous distalsomes, and large vacuoles.

In this paper, we demonstrate the kinetics of uptake of the dye F M 4-64 via the plasma membrane of the growing germ tube and its migration within regions I and II. We show that the dye first accumulates in the subapical region. Subsequently it is redistributed and then labels the area with apical vesicles in the apex or the prenuclear area of region II. Our results present the first evidence for endocytosis in a fungal germ tube.

\section{MATERIAL AND METHODS}

\section{Fungal Material}

Uromyces fabae (Grev.) de Bary ex F uckel was propagated on broad bean, Vicia faba L. cv. Con Amore, in a greenhouse at $20 \pm 5^{\circ} \mathrm{C}$. U redospores were collected 14 days after inoculation and stored up to 1 week at $4^{\circ} \mathrm{C}$ before use. Alternatively, spores were stored at $-70^{\circ} \mathrm{C}$ after harvest. Aliquots of frozen spores were thawed and used immediately. Twenty to thirty milligrams of spores was dusted within a settling tower and allowed to settle on coverslips. Subsequently, spores were misted with deionized water and incubated in darkness at $100 \% \mathrm{RH}$ for $3 \mathrm{~h}$ at $20 \pm 0.5^{\circ} \mathrm{C}$.

\section{Video Microscopy}

A Standard microscope (Carl Zeiss, Jena, G ermany) was equipped with phase contrast (100×; N eofluar; NA 1.3), oil immersion condensor lens (NA 1.4), and epifluorescence (excitation $450-490 \mathrm{~nm}$, emission $\leq 520 \mathrm{~nm}$ ) with a current stabilized 100-W HBO lamp. The microscope was connected to a Proxicam H L 5 intensified charge-coupled device camera and analogous enhancer unit (Proxitronic, Bensheim, Germany) for controlling gain and black level. The configuration was left unaltered throughout the period of observation. The signal was passed to a Power $M$ acintosh 7600 computer equipped with a frame grabber card (AV quicktime digitizer, $768 \times 576$ pixels with 8 bits per pixel). The mouse of the computer was connected to a time-regulated shutter in the light path of the excitation lamp. A mouse click opened this shutter and frames were captured.

\section{Measuring Fluorescence Dynamics}

To measure fluorescence by germ tubes during the first $10 \mathrm{~s}$, the shutter was left open and every $0.5 \mathrm{~s}$, one frame was captured. To follow dye uptake over a period of 20 min, the shutter was opened every $60 \mathrm{~s}$ for $0.25 \mathrm{~s}$ and one frame was captured. There was no evidence of photobleaching, even when exposure times were doubled.

\section{Image Processing and Analysis}

Images were processed and analyzed using the public domain I mage software written by Wayne Rasband at $\mathrm{N} \mathrm{IH}$ (NIH Image available via FTP transfer from zippy.nimh. nih.gov). Captured images were smoothed three times with the integrated smoothing filter. Fluorescence of the membrane or the cytosol was measured over selections as indicated in the figures. The plot profiles indicate fluorescence emission in gray values $(0=$ black, $255=$ red). Individual images were arranged and labeled with Adobe Photoshop 4.0 and printed with a F ujix Pictography 3000 digital printer (F uji Photo F ilm G .m.b.H ., D üsseldorf, Germany).

\section{Specimen Preparation}

Coverslips with the germinating spores were laid upside down on slides with a spacer of $0.5 \mathrm{~mm}$ thickness. Water, $0.2 \mathrm{ml} / \mathrm{min}, 22^{\circ} \mathrm{C}$, was added to one side of the coverslip and sucked away from the other side.

\section{Dye Application}

The vital dye $\mathrm{N}$-(3-triethylammonium propyl)-4-(pdiethylaminophenylhexatrienyl) pyridinium dibromide, FM 4-64, was obtained from M olecular Probes, Inc. (E ugene, OR). A stock solution, $16 \mathrm{mM}$ in dimethyl sulfoxide, was diluted 1:4000 with water. Fifty microliters of this dilution was added to one side of the coverslip and sucked away from the other side. When the dye reached the hyphae under observation, the experiment started (time 0 ).

\section{Inhibition Experiments}

For inhibition experiments, the germ tubes were exposed to $5 \mathrm{mM} \mathrm{NaN}_{3}$ for $1 \mathrm{~min}$ and subsequently to the dye (including the inhibitor) as described. Alternatively, the microscope stage was cooled with a recirculator; the slide and the objective lens were cooled by lowering the room temperature. The temperature of the water under the coverslip, $4^{\circ} \mathrm{C}$, was controlled with a thermocouple (Conrad-E lektronik, H irschau, G ermany). 


\section{Statistical Analysis}

$D$ ata required for determining mean values and standard deviations were imported into an EXCEL spreadsheet and evaluated as described by Reynaga-Pena et al. (1997). The areas of fluorescence measured are shown in the figures.

\section{RESULTS}

\section{Organization of the Germ Tube}

The germ tubes of $U$. fabae exhibited an organization similar to those of U. phaseoli (H och and Staples, 1985). $\mathrm{M}$ ost conspicuous were the two nuclei, located in region III, about 45-60 $\mu \mathrm{m}$ from the tip (Fig. 1). Between the hyphal tip and the two nuclei, the light microscope did not exhibit much differentiation of the cytoplasm except for the utmost tip area, which includes the first $5 \mu \mathrm{m}$. U sing interference-contrast microscopy, this area looked blank (Fig. 1). Phase-contrast optics, however, revealed a diffuse dark cloud within the apical pole (F ig. 2). The diameter of the cloud was $2.5 \pm 0.23 \mu \mathrm{m}(\mathrm{n}=34)$. Sometimes filamentous structures, probably mitochondria, reached into the apex. Tapping the coverslip made the cloud disappear (F ig. 3). Subsequently, the mitochondria migrated into the tip area which seemed to round up (F ig. 4). Thus, fungal growth was interrupted.

D uring growth of the hypha, the dark cloud in the apex displayed movements which preceded and seemed to determine the subsequent growth direction of the tip. Figures 5-7 show a cloud in the left part of the hyphal tip and a subsequent bulging of the tip wall into this direction. At $22^{\circ} \mathrm{C}$, hyphae grew at a speed of $1.1 \pm 0.5 \mu \mathrm{m} / \mathrm{min}$ $(n=10)$.

\section{Uptake of the Dye FM4-64}

No fluorescence of germ tubes was observed at the start of the experiment. Within $4 \mathrm{~s}$ of dye contact with the germ tube, fluorescence along the germ tube periphery could be detected ( $\mathrm{Fig}$. 8). F luorescence increased gradually ( $\mathrm{Fig}$. 9) and reached a peak after 1 min (Fig. 10), with the highest intensity at the hyphal tip. Subsequently, fluorescence of hyphal contours decreased and the cytoplasm

\section{region I region II region III region $\mathrm{IV}+\mathrm{V}$}
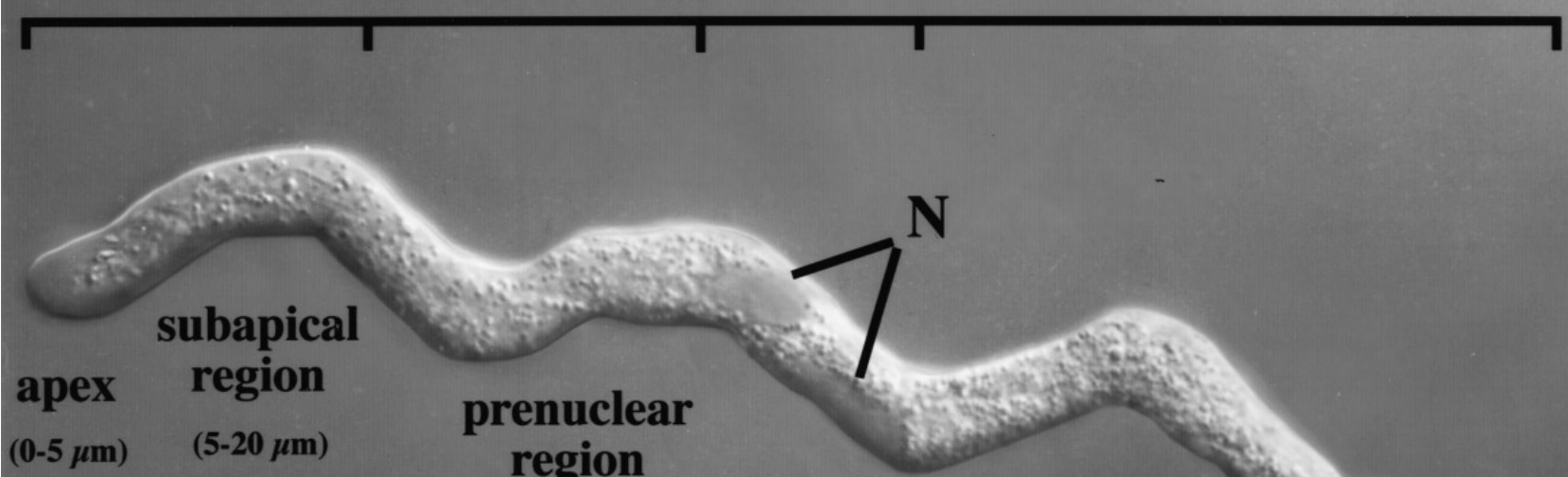

$(0-5 \mu \mathrm{m}) \quad(5-20 \mu \mathrm{m})$

prenuclear region

(35-45 $\mu \mathrm{m})$

FIG. 1. Light micrograph of the $U$. fabae germ tube, interference contrast optics. Bar, $5 \mu \mathrm{m}$. 

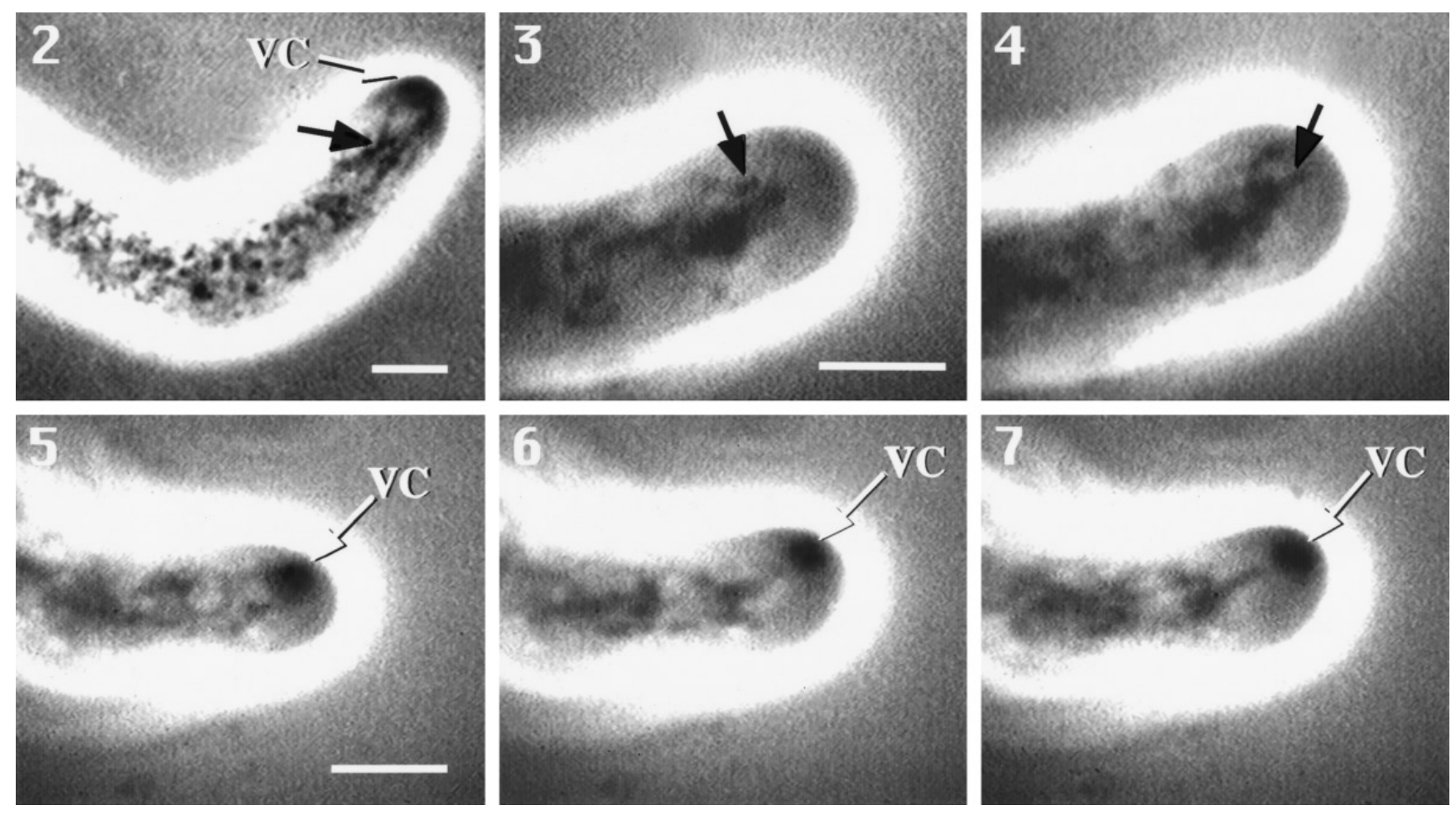

FIGS. 2-7. Phase-contrast observation revealed a dark cloud (VC-vesicle cloud) in the apex and filamentous structures (mitochondria) behind (black arrow) aligned in the longitudinal axis of the germ tube (Fig. 2). The dark cloud had disappeared about 2 min after tapping the coverslip (Fig. 3). Subsequently, filamentous structures (black arrow) migrated into the apex within $5 \mathrm{~min}$ (F ig. 4). Series of pictures showing movements of the dark cloud in the apex during hyphal growth. If the cloud moved from the center of the apex (F ig. 5) to the left, the hypha bulged on the left side (F ig. 6). Subsequently, germ tube growth followed the position of the cloud (F ig. 7). Bars, $5 \mu \mathrm{m}$.

started to be labeled. About 5 min after the start of the experiment, an area of the cytoplasm reaching from 5 to 20 $\mu \mathrm{m}$ of the hyphal tip exhibited intense fluorescence ( $\mathrm{Fig}$. 11). Fifteen minutes after start, fluorescence along the hyphal wall had decreased to a constant level and fluorescence in the center of the hyphal apex appeared (Fig. 12). It was a small, round zone of fluorescence, similar to the dark cloud seen with phase-contrast optics, but more distinct and smaller in diameter $[1.9 \pm 0.24 \mu \mathrm{m}(n=27)]$. An evaluation of fluorescence measurements from 10 hyphae revealed the first evidence of fluorescence of the round zone within the apex al ready $10 \mathrm{~min}$ after start ( $\mathrm{Fig}$. 15D). The center of the dark cloud observed with phase contrast (F ig. 16) and the center of the round fluorescent zone ( $\mathrm{F}$ ig. 17) were identical.

Twenty minutes after start, a prenuclear area of region II exhibited bright fluorescence ( $F$ ig. 13). It extended from 35 to $45 \mu \mathrm{m}$ of the hyphal tip, close to the two nuclei. F luorescence in the round zone of the apex did not change in intensity during these last $10 \mathrm{~min}$ of the experiment.
This was in contrast to the fluorescence along the hyphal wall, which, after having reached the maximum (after 1 $\mathrm{min}$ ), declined gradually and reached an even, low level after $15 \mathrm{~min}$ (F ig. 14). D ecrease of fluorescence occurred at a similar rate at the apex, in region I and in region II.

In the presence of the dye, hyphae grew at a speed of $1.0 \pm 0.3 \mu \mathrm{m} / \mathrm{min}(n=10)$. Fluorescence intensities differed considerably in experiments performed at 22 or $4^{\circ} \mathrm{C}$ or in the presence of $5 \mathrm{mM} \mathrm{NaN}_{3}$ (Figs. 15A-15C). In both controls $\left(4^{\circ} \mathrm{C}, \mathrm{NaN}_{3}\right)$, fluorescence along hyphal contours reached the typical peak values, but did not decrease during the course of the experiment. The cytoplasm remained unlabeled.

\section{DISCUSSION}

To study endocytosis in fungi, we lack the morphological and biochemical approaches that are well established in 


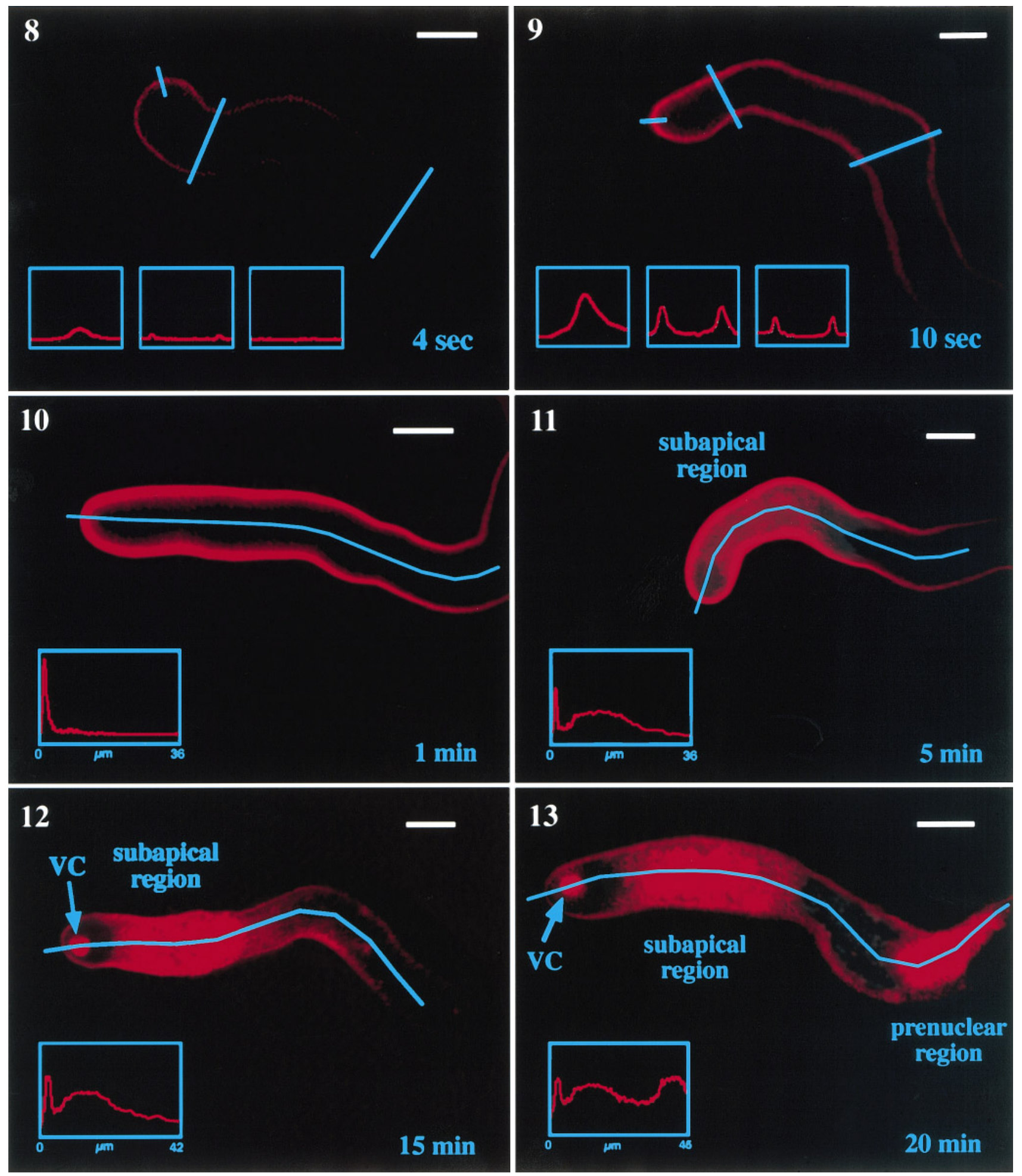

FIGS. 8 AND 9. Shortly after exposure of germ tubes to F M 4-64 (Fig. 8, 4 s), a signal could be measured. The highest intensity of fluorescence was observed in the apical dome (F ig. 9, $10 \mathrm{~s}$ ). Inserts show the relative increase of fluorescence measured along the bars positioned over the hypha. Bars, $5 \mu \mathrm{m}$. FIGS. 10-13. The maximum of membrane fluorescence was reached after $1 \mathrm{~min}$ (F ig. 10). F ive min after the start of the experiment, a subapical region in the hyphal cytoplasm showed fluorescence (F ig. 11). Ten minutes later, a well-defined round zone (VC - vesicle cloud) in the hyphal apex (F ig. 12) and, another 5 min later, the prenuclear region exhibited bright fluorescence (F ig. 13). Inserts show the relative amount of fluorescence measured along the line positioned over the long axis of the hypha and median. Bars, $5 \mu \mathrm{m}$. 


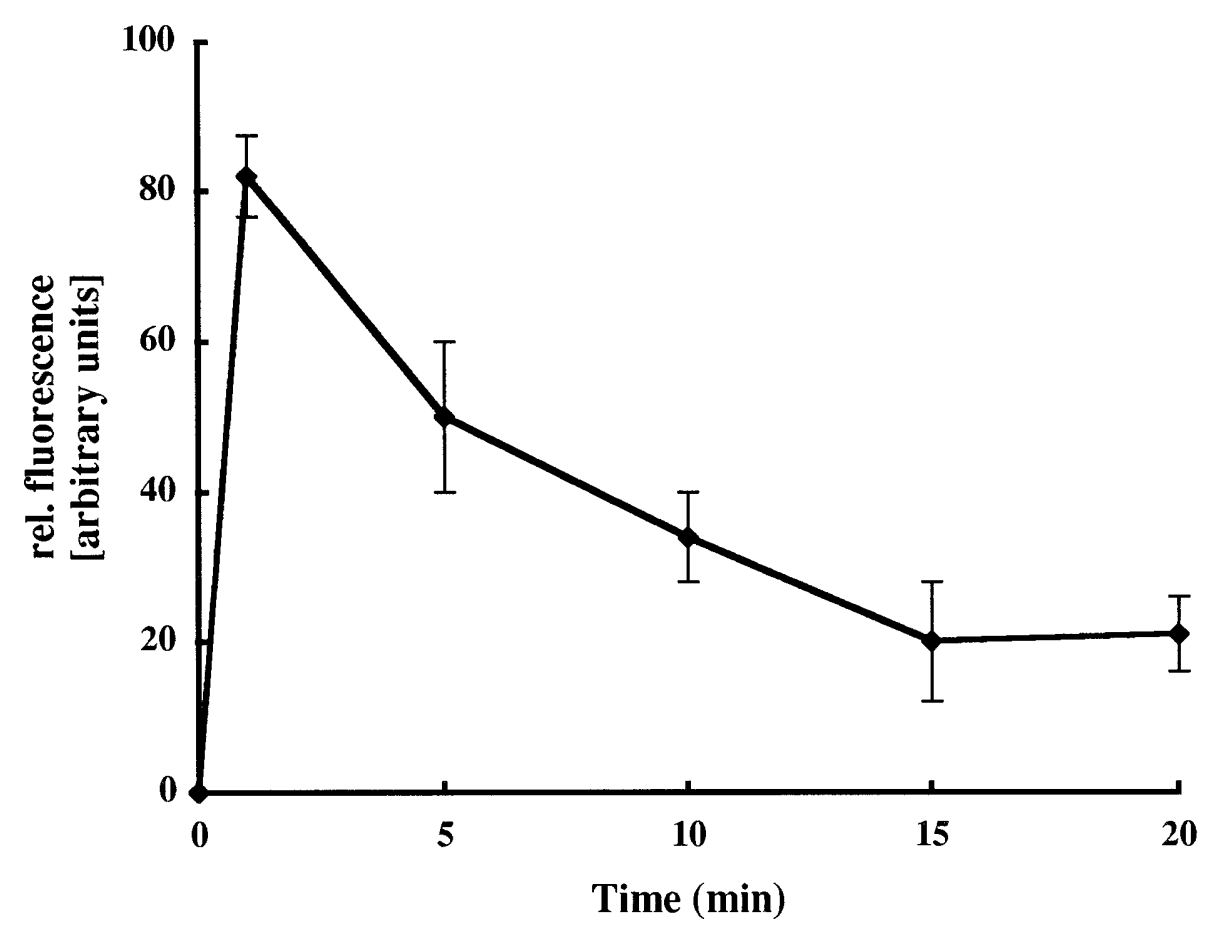

FIG. 14. Kinetics of fluorescence along the hyphal contours, measured $5 \mu \mathrm{m}$ behind the apical pole during a 20-min period.

animal systems (M ellman, 1996; Robinson et al., 1996; Salzman and Maxfield, 1993). S. cerevisiae does not internalize most ligands generally recommended as tracers to visualize intermediates between the plasma membrane and the vacuole. Also, no biochemical assay for any endocytotic fusion or transport event has been performed with yeast membranes in vitro (Vida and E mr, 1995). A similar situation exists with fungal hyphae, where membrane-impermeant probes either did not penetrate fungal walls or were not internalized by fluid-phase endocytosis (Cole et al., 1997).

F M 1-43 and other styryl dyes have been used successfully to monitor endocytosis, exocytosis, and the corresponding endosomal traffic in numerous animal cells (Betz et al., 1992; $\mathrm{H}$ enkel et al., 1996). The ionically charged heads and hydrophobic tails of these dyes suggest the following mechanism of action: Dye molecules partition from the surrounding bathing solution into the outer leaflet of all surface membranes but cannot penetrate into the cytoplasm. Once trapped by the membrane, fluorescence increases. As soon as the dye-labeled membrane is internalized, for example, by endocytosis, the dye labels the resulting endosome. In Saccharomyces, FM 4-64 initially stains the plasma membrane, then small, punctate cytoplasmic compartments, and finally the vacuolar membrane
(Rieder et al., 1996). Mutants defective in ER to Golgi transport (sec 18), Golgi transport (sec 14), or receptormediated endocytosis (end 3 and end 4) did not completely lack vacuolar membrane labeling. $\mathrm{H}$ owever, $\mathrm{NaN}_{3} / \mathrm{N} \mathrm{aF}$ treatment inhibited any vacuolar staining, suggesting that membrane delivery of FM 4-64 from the plasma membrane to the vacuole was energy dependent. In addition, uptake was temperature dependent and intermediate structures could be trapped by temperature shifts (Vida and $E \mathrm{mr}, 1995)$. These authors suggest that FM 4-64 staining of the vacuole is mediated by endocytotic uptake of the dye and vesicle-mediated transport via endosomelike intermediates. Probably this represents the natural pathway for endocytosis of endogenous plasma membrane lipids and proteins. However, a non-fluid-phase uptake mechanism cannot be excluded (Stack et al., 1995).

Similar to the situation in Saccharomyces, the germ tube membrane of U romyces immediately exhibits fluorescence after incubation with FM 4-64. We cannot explain the difference in plasma membrane fluorescence along the germ tube, with the highest intensity in the apex and barely any fluorescence in the hyphal area behind the two nuclei. E ither the membrane has very different endocytotic activities in the different areas of the growing hypha or the fungal wall becomes impermeable to the dye molecules as 

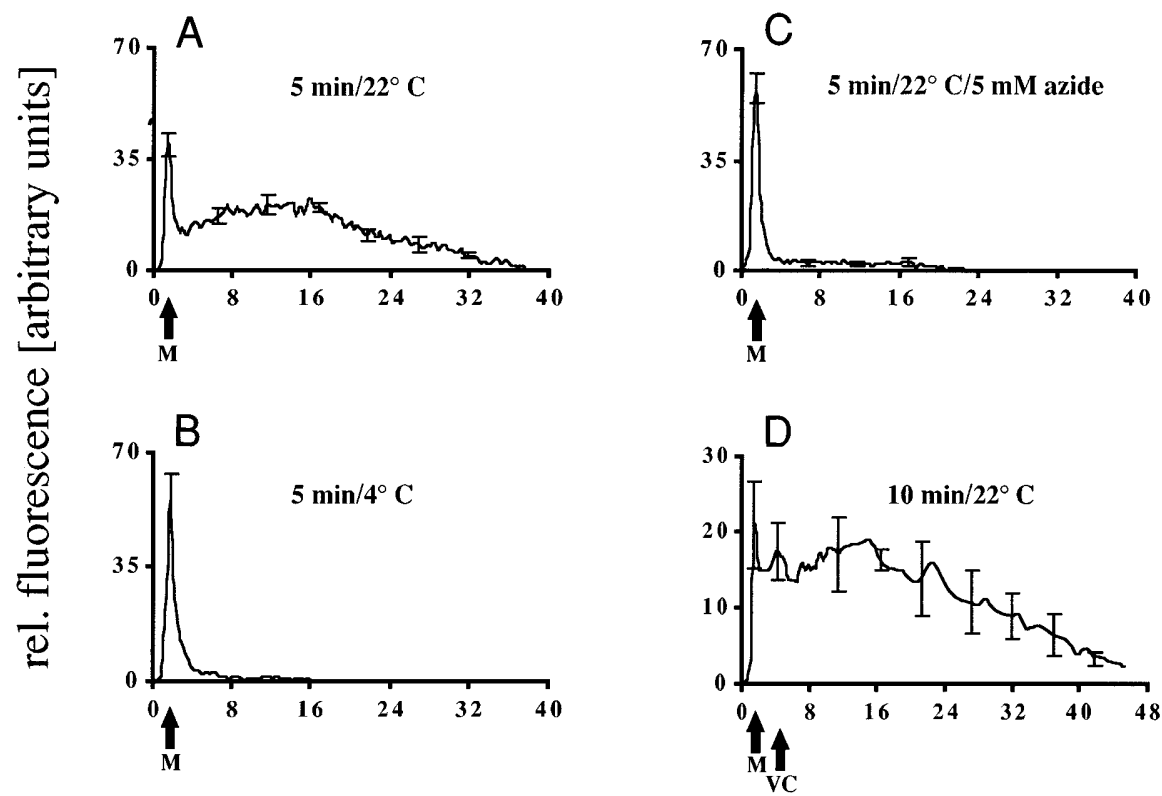

length of line selection $[\mu \mathrm{m}]$

FIG. 15. M easurements of fluorescence intensity along a line positioned over the long axis of the hypha (M - presumed plasma membrane, VC - vesicle cloud). (A) Fluorescence of the apical membrane and of the cytoplasm after $5 \mathrm{~min}$. (B) Control experiment performed at $4^{\circ} \mathrm{C}$. (C) $\mathrm{Control}$ experiment performed in the presence of $5 \mathrm{mM} \mathrm{N}$ aN $_{3}$. (D) F luorescence $10 \mathrm{~min}$ after the start of the experiment, revealing the first faint label in the vesicle cloud zone. Standard errors of selected time points are shown (means of 8-10 hyphae each).

it ages. M easurements of pore sizes in walls which exist for O omycetes (Money, 1990) or for plants (Carpita et al., 1979) are missing for U romyces.

The redistribution of fluorescence from the contours of the hypha, probably the plasma membrane, to different parts of the germ tube cytoplasm confirm observations of organelle distribution within the bean rust germ tube (Hoch and Staples, 1983). The authors found an abundance of $\mathrm{G}$ olgi bodies and highly variable vesicles in region I. This area corresponds, except for the apex, to what we call the subapical region (5-20 $\mu \mathrm{m}$ from the apex). Here, the earliest cytoplasmic fluorescence was observed. We
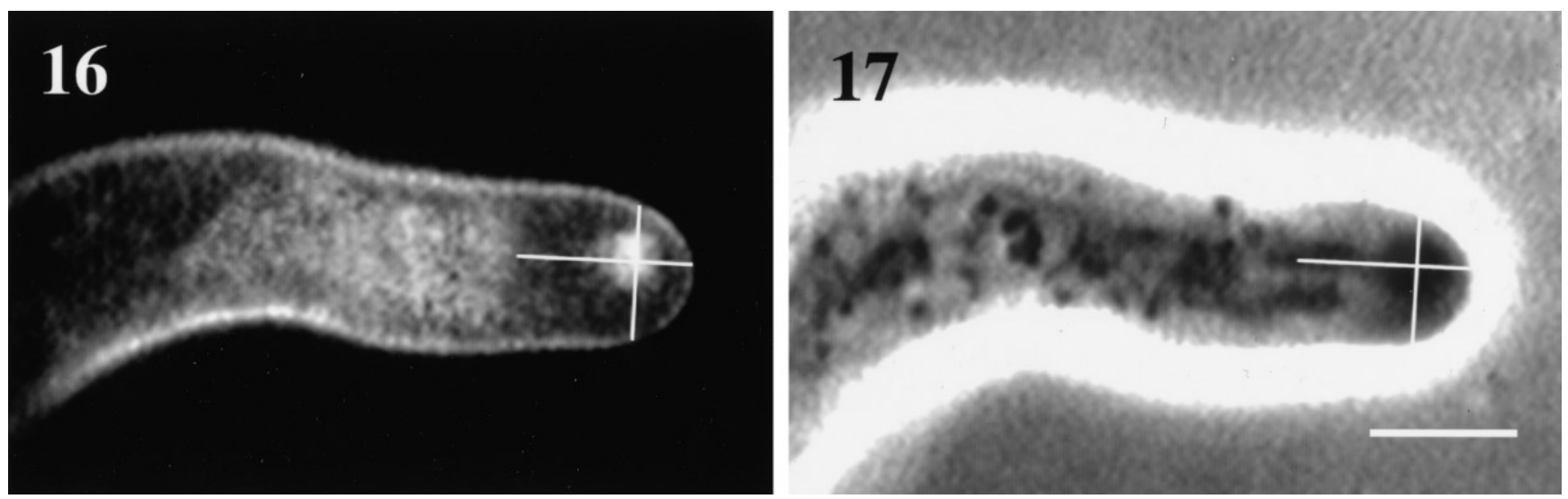

FIGS. 16 AND 17. H yphal tip 15 min after feeding with F M 4-64, exhibiting bright fluorescence in the middle of the apex. The same hypha as in F ig. 16 was shot only a few seconds later with phase-contrast optics. The positions of the crosses in F igs. 16 and 17 are identical. Bar, $5 \mu \mathrm{m}$. 
suggest that this subapical region contains an intermediate compartment with endosomes or similar structures.

The next area that exhibits fluorescence is a round zone corresponding to the dark cloud visible with phase-contrast optics in the apex of the hyphal tip. The position of this dark cloud corresponds to the position of apical vesicles observed in Uromyces appendiculatus after conventional fixation (Littlefield and Heath, 1979) or after freeze substitution (H och et al., 1987). The cloud in the apex of $U$. fabae appears less discrete compared to the small granule called Spitzenkörper observed in hyphal tips of many fungi (Grove and Bracker, 1970) and does not fit into any of the eight morphological patterns of Spitzenkörper organization described by Lopez-F ranco and Bracker (1996). The cloud observed in the apex of the $U$. fabae germ tube has more similarities to the apical vesicles of pollen tubes (Derksen, 1996; Larson, 1965), rhizoids of some algae (Sievers, 1967), budding yeasts (M oor, 1967), or Phytophthora infestans (Förster and M endgen, 1987). H owever, the movements of the apical vesicles during hyphal elongation and their correlation with the growth direction are very similar to observations with the Spitzenkörper (Reynaga-Pena et al., 1997). Therefore, it is difficult to discriminate the dark cloud from the Spitzenkörper observed by Girbardt (1957) with the light microscope. It may be coincident with the vesicle supply center from where vesicles are assumed to migrate to the apical plasma membrane (Bartnicki-G arcia et al., 1989).

Finally, some 20 min after the start of the experiment, region II of the cytoplasm is stained. Hoch and Staples (1983) observed numerous small vacuoles in this region, which seem to belong to the lysosomal system of the cell. F eeding hyphae with 6-carboxyfluorescein diacetate exhibited bright fluorescence in this area (unpublished result).

Our results suggest the following sequence of events: The dye appears to enter the cytoplasm along the hypha by fluid-phase endocytosis in an energy-dependent manner since $\mathrm{NaN}_{3}$ or low temperature inhibits this process. At first, the dye accumulates in endosomal compartments of the subapical region. Subsequently the dye is either redistributed to the apex from where it is secreted via the cloud of apical vesicles or it accumulates in a lysosomal compartment close to the nuclei. The turnover time of the labeled membrane, i.e., the time between uptake of the dye and secretion of the dye at the apex via the cluster of apical vesicles, amounts to about $15 \mathrm{~min}$. Afterward, membrane fluorescence at the hyphal tip reaches a steady state due to a balance between loading and depletion of the membrane with dye by secretory and endocytotic events.

\section{ACKNOWLEDGMENTS}

We thank Dr. Christine Struck, D r. M atthias Hahn, and D r. Stefan Wirsel and two anonymous reviewers for reading the manuscript. The D eutsche $F$ orschungsgemeinschaft supported this project (M e 523/15).

\section{REFERENCES}

Bartnicki-Garcia, S., H ergert, F., and Gierz, G. 1989. Computer simulation of morphogenesis and the mathematical basis for hyphal (tip) growth. Protoplasma 153: 46-57.

Betz, W. J., M ao, F., and Bewick, G. S. 1992. Activity-dependent fluorescent staining and destaining of living vertebrate motor nerve. J. Neurosci. 12: 363-375.

Carpita, N ., Sabularse, D., M ontezinos, D ., and Delmer, D. P. 1979. Determination of the pore size of living plant cells. Science 205: 1144-1147.

Cole, L., H yde, G. J., and Ashford, A. E . 1997. U ptake and compartmentalization of fluorescent probes by Pisolithus tinctorius hyphae: $\mathrm{E}$ vidence for an anion transport mechanism at the tonoplast but not for fluid-phase endocytosis. Protoplasma 199: 18-29.

Collinge, A. J., and Trinci, A. P. 1974. Hyphal tips of wild-type and spreading colonial mutants of Neurospora crassa. Arch. Microbiol. 99: 353-368.

D erksen, J. 1996. Pollen tubes: A model system for plant cell growth. B ot. Acta 109: 341-345.

F örster, H ., and M endgen, K. 1987. I mmunocytochemical localization of pectin esterases in hyphae of Phytophthora infestans. Can. J. Bot. 65: 2607-2613.

Girbardt, M. 1957. Der Spitzenkörper von Polystictus versicolor (L.). Planta 50: 47-59.

Gow, N. A. R. 1989. Control of the extension of the hyphal apex. In Current Topics in Medical Mycology 3 (M. R. McGinnis and M. Borgers, E ds.), pp. 109-152. Springer, N ew York.

Grove, S. N., and Bracker, C. E. 1970. Protoplasmic organization of hyphal tips among fungi: Vesicles and Spitzenkörper. J. Bacteriol. 104: 989-1009.

H eath, I. B., and H eath, M. C. 1979. Structural studies of the development of infection structures of cowpea rust, Uromyces phaseoli var. vignae. II. Vacuoles. Can. J. Bot. 57: 1830-1837.

H enkel, A. W., L uebke, J., and B etz, W. J. 1996. F M 1-43 dye ultrastructural localization in and release from frog motor nerve terminals. Proc. Natl. Acad. Sci. USA 93: 1918-1923.

Heuser, J., Zhu, Q., and Clarke, M. 1993. Proton pumps populate the contractile vacuoles of Dictyostelium amoebae. J. Cell Biol. 121: 1311-1327.

H och, H. C., and Staples, R. C. 1983. Ultrastructural organization of the non-differentiated uredospore germling of Uromyces phaseoli variety typica. M ycologia 75: 795-824.

H och, H. C., and Staples, R. C. 1985. The microtubule cytoskeleton in hyphae of Uromyces phaseoli germlings: I ts relationship to the region of nucleation and to the $\mathrm{F}$-actin cytoskeleton. Protoplasma 124: 112-122. 
H och, H . C., Tucker, B. E ., and Staples, R. C. 1987. An intact microtubule cytoskeleton is necessary for mediation of the signal for cell differentiation in Uromyces. Eur. J. Cell Biol. 45: 209-218.

Larson, D. A. 1965. Fine structural changes in the cytoplasm of germinating pollen. Am. J. Bot. 52: 139-154.

Littlefield, L. J., and Heath, M. 1979. Ultrastructure of Rust Fungi. Academic Press, N ew York.

L opez-F ranco, R., and Bracker, C. E . 1996. D iversity and dynamics of the Spitzenkörper in growing hyphal tips of higher fungi. Protoplasma 195: 90-111.

M ellman, I. 1996. Endocytosis and molecular sorting. Annu. Rev. Cell Biol. 12: 575-625.

M oney, N. P. 1990. M easurements of pore size in the hyphal cell wall of Achlya bisexualis. Exp. M ycol. 14: 234-242.

M oor, H . 1967. Endoplasmic reticulum as the initiator of bud formation in yeast (S. cerevisiae). Arch. M icrobiol. 57: 135-146.

Reynaga-Pena, C. G., Gierz, G ., and B artnicki-Garcia, S. 1997. Analysis of the role of the Spitzenkörper in fungal morphogenesis by computer simulation of apical branching in Aspergillus niger. Proc. Natl. Acad. Sci. USA 94: 9096-9101.

Rieder, S. E ., Banta, L. M ., Koehrer, K., M cCaffery, J. M ., and E mr, S. D.
1996. M ultilamellar endosome-like compartment accumulates in the yeast vps28 vacuolar protein sorting mutant. M ol. Biol. C ell 7: 985-999.

Riezman, H. 1997. Molecular mechanisms of endocytosis. Cell 91: 731-738.

Robinson, D. G., and Hillmer, S. 1990. Endocytosis in plants. Physiol. Plant. 79: 96-104.

Robinson, M. S., Watts, C., and Zerial, M. 1996. M embrane dynamics in endocytosis. Cell 84: 13-21.

Salzman, N. H., and Maxfield, F. R. 1993. Quantitative fluorescence technique for the characterization of endocytosis in intact cells. Subcell. Biochem. 19: 95-123.

Sievers, A. 1967. E lektronenmikroskopische U ntersuchungen zur geotropischen Reaktion. II. D ie polare Organisation des normal wachsenden Rhizoids von Chara foetida. Protoplasma 64: 225-253.

Stack, J. H., H orazdovsky, B., and E mr, S. D. 1995. Receptor-mediated protein sorting to the vacuole in yeast: Roles for a protein kinase and GTP-binding proteins. Annu. Rev. Cell Biol. 11: 1-33.

Vida, T. A., and E mr, S. D. 1995. A new vital stain for visualizing vacuolar membrane dynamics and endocytosis in yeast. J. Cell Biol. 128: 779-792. 